Physics

Physics Research Publications

Purdue University

Year 2004

\title{
Electron-phonon renormalization of electronic band gaps of semiconductors: Isotopically enriched silicon
}
S. Tsoi*
H. Alawadhi ${ }^{\dagger}$
X. $\mathrm{Lu}^{\ddagger}$
J. W. Ager**
C. Y. Liao ${ }^{\dagger \dagger}$
H. Riemann ${ }^{\ddagger \ddagger}$
E. E. Haller ${ }^{\S}$
S. Rodriguez
A. K. Ramdas"

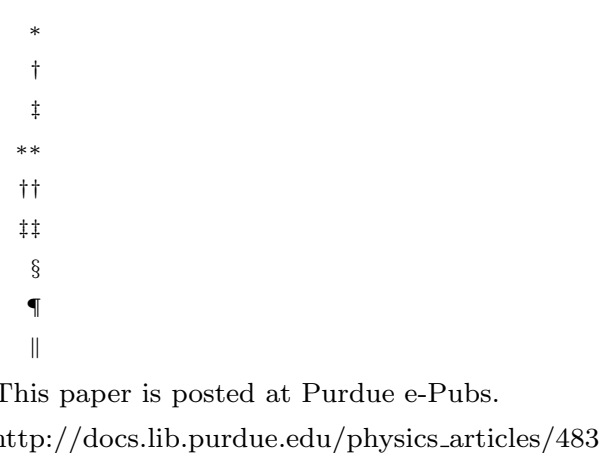




\title{
Electron-phonon renormalization of electronic band gaps of semiconductors: Isotopically enriched silicon
}

\author{
S. Tsoi, ${ }^{1}$ H. Alawadhi, ${ }^{2}$ X. Lu, ${ }^{1}$ J. W. Ager III, ${ }^{1}$ C. Y. Liao, ${ }^{3,4}$ H. Riemann, ${ }^{5}$ E. E. Haller,${ }^{3,4}$ S. Rodriguez,${ }^{1}$ and \\ A. K. Ramdas ${ }^{1}$ \\ ${ }^{1}$ Department of Physics, Purdue University, West Lafayette, Indiana 47907, USA \\ ${ }^{2}$ Department of Basic Sciences, University of Sharjah, United Arab Emirates \\ ${ }^{3}$ Lawrence Berkeley National Laboratory, Berkeley, California 94720, USA \\ ${ }^{4}$ Department of Materials Science and Engineering, University of California at Berkeley, Berkeley, California 94720, USA \\ ${ }^{5}$ IKZ, Berlin, Germany
}

(Received 25 June 2004; published 3 November 2004)

\begin{abstract}
Photoluminescence and wavelength-modulated transmission spectra displaying phonon-assisted indirect excitonic transitions in isotopically enriched ${ }^{28} \mathrm{Si},{ }^{29} \mathrm{Si},{ }^{30} \mathrm{Si}$, as well as in natural $\mathrm{Si}$, have yielded the isotopic mass $(M)$ dependence of the indirect excitonic gap $\left(E_{g x}\right)$ and the relevant phonon frequencies. Interpreting these measurements on the basis of a phenomenological theory for $\left(\partial E_{g x} / \partial M\right)$, we deduce $E_{g x}(M=\infty)$ $=(1213.8 \pm 1.2) \mathrm{meV}$, the purely electronic value in the absence of electron-phonon interaction and volume changes associated with anharmonicity.
\end{abstract}

DOI: 10.1103/PhysRevB.70.193201

The electronic band structure of the tetrahedrally coordinated elemental group IV and the compound III-V and II-VI semiconductors have been experimentally delineated and theoretically calculated with exceptional completeness. ${ }^{1} \mathrm{Un}-$ til recently, the experimental determinations of the band gaps of these semiconductors were made on specimens whose isotopic composition reflected the elemental natural abundance. The situation in this regard changed dramatically with access to monoisotopic $\mathrm{Ge}$ in the late $1980 \mathrm{~s},{ }^{2}$ making possible the growth of large, isotopically engineered, single crystals. Isotopically controlled ${ }^{12} \mathrm{C}_{1-x}{ }^{13} \mathrm{C}_{x}$ single crystals of diamond have been grown over the entire composition range. ${ }^{3}$ Only recently, ${ }^{4}$ a similar achievement has been repeated with $\mathrm{Si}$ and single crystal isotopically enriched ${ }^{28} \mathrm{Si},{ }^{29} \mathrm{Si}$, and ${ }^{30} \mathrm{Si}$ as well as isotopic alloys with a controlled $M$, the average resultant atomic mass, have been grown.

The changes in the band gaps $\left(E_{G}\right)$ of semiconductors as a function of the thermodynamic variables pressure $(P)$ and temperature $(T)$ have been a subject of comprehensive investigations since the early days of semiconductor physics. ${ }^{5}$ With the advent of isotopically controlled specimens, their dependence on the average atomic mass can now be experimentally addressed. The variation of $E_{G}$ as a function of $M$ is expressed as ${ }^{6}$

$$
\left(\frac{\partial E_{G}}{\partial M}\right)_{T, P}=\left(\frac{\partial E_{G}}{\partial M}\right)_{T, V}+\frac{D}{V}\left(\frac{\partial V}{\partial M}\right)_{T, P} .
$$

Here, $D=-B\left(\partial E_{G} / \partial P\right)_{T, M}$ is the deformation potential for hydrostatic stress and the band gap in question; and $B$, the bulk modulus. The first term on the right-hand side of Eq. (1) reflects the variation of $E_{G}$ with $M$, at given temperature and volume, due to electron-phonon interaction with contributions linear and quadratic in atomic displacements $u_{\mathbf{n}}(\mathbf{q}, j)$, the former taken in second order perturbation (Fan terms ${ }^{7}$ ) and the latter in first order (Debye-Waller terms ${ }^{8}$ ); $\mathbf{n}$ denotes the position of an atom, $\mathbf{q}$ the wave vector of a phonon of branch $j$. The resultant variation in $E_{G}$ is propotional to ther-
PACS number(s): 78.55.Ap, 63.20.Kr, 78.40.Fy

mal averages $\left\langle u_{\mathbf{n}}^{2}(\mathbf{q}, j)\right\rangle$, which equal $\left(\hbar / M \omega_{\mathbf{q} j}\right)\left(\bar{n}_{\mathbf{q} j}+\frac{1}{2}\right), \bar{n}_{\mathbf{q} j}$ being the Bose-Einstein occupation number. The use of the average mass is implicit in the virtual crystal approximation in the lattice dynamics of crystals with random isotopic disorder. At $T=0 \mathrm{~K},\left\langle u_{\mathbf{n}}^{2}(\mathbf{q}, j)\right\rangle=\left(\hbar / 2 M \omega_{\mathbf{q} j}\right)$, the zero-point contribution. Thus, both the Fan and the Debye-Waller contributions to the change in $E_{G}$ are proportional to $M^{-1 / 2}$ under the assumption that the phonon frequencies scale as the inverse square root of the average mass. However, at high temperatures $\left\langle u_{\mathbf{n}}^{2}(\mathbf{q}, j)\right\rangle \approx\left(k_{B} T / M \omega_{\mathbf{q} j}^{2}\right)$ becomes independent of $M$ and the isotopic effects should progressively become less important with increasing temperature. The second term in Eq. (1) results from the change in volume arising from the difference in average atomic mass. Hu et al. ${ }^{9}$ have shown recently that, at $T=0 \mathrm{~K}$,

$$
\frac{1}{V}\left(\frac{\partial V}{\partial M}\right)_{T}=-\frac{\sqrt{6} B^{\prime} \hbar}{a^{5 / 2} B^{1 / 2} M^{3 / 2}},
$$

where $a$ is the lattice parameter defined by the cubic unit cell of $\mathrm{Si}$ and $B^{\prime}=(\partial B / \partial P)_{T}$, the third order bulk modulus. Note, the change in $E_{G}$ due to volume as a function of $M$, like that due to electron-phonon interaction, is proportional to $M^{-1 / 2}$.

In this paper we report on the phonon-assisted indirect transitions in nominally monoisotopic single crystals of ${ }^{28} \mathrm{Si}$, ${ }^{29} \mathrm{Si}$, and ${ }^{30} \mathrm{Si}$ as well as in $\mathrm{Si}$ with natural isotopic abundance (see Table I) observed in photoluminescence (PL) and wavelength-modulated transmission (WMT).

As is well known, the indirect transitions between the $\Gamma_{8}^{+}$ zone center valence band maximum and the $\Delta_{1}$ conduction band minima along $\langle 100\rangle$ of $\mathrm{Si}$, observed in PL and absorption, occur with the emission or absorption of wave-vectorpreserving optical and acoustic phonons having q's equal to that of $\Delta_{1}$; at low temperatures, the indirect transitions are assisted by phonon emission. Thus, their signatures in PL occur at $E_{g x}-\hbar \omega_{\mathbf{q}, j}$, where $E_{g x}$ is the excitonic band gap and $j$ corresponds to transverse acoustic (TA), longitudinal acoustic (LA), longitudinal optical (LO), and transverse 
TABLE I. Isotopic composition and average masses of isotopically enriched Si samples.

\begin{tabular}{lcccc}
\hline \hline & $\%^{28} \mathrm{Si}$ & $\%^{29} \mathrm{Si}$ & $\%^{30} \mathrm{Si}$ & $M$ \\
\hline Sample ${ }^{28} \mathrm{Si}$ & 99.92 & 0.075 & 0.005 & 27.98 \\
Sample ${ }^{N a t} \mathrm{Si}$ & 92.23 & 4.67 & 3.1 & 28.09 \\
Sample ${ }^{29} \mathrm{Si}$ & 4.3 & 91.37 & 4.3 & 28.98 \\
Sample ${ }^{30} \mathrm{Si}$ & 4.29 & 7.46 & 88.25 & 29.81 \\
\hline \hline
\end{tabular}

optical (TO) phonons. In a similar fashion, the indirect thresholds in absorption, revealed as derivative signatures in, say, WMT, are expected at $E_{g x}+\hbar \omega_{\mathbf{q}, j}$. A combination of PL and WMT thus yields both $E_{g x}$ and $\hbar \omega_{\mathbf{q}, j}$ as functions of $M{ }^{6}$

The PL spectra of the monoisotopic and natural $\mathrm{Si}$ are displayed in Fig. 1(a) at $T=20 \mathrm{~K}$ and in Fig. 1(b) for $T$ $=9 \mathrm{~K}$. The luminescence was excited with $500 \mathrm{~mW}$ of all lines from an $\mathrm{Ar}^{+}$laser focused to a spot $200 \mu \mathrm{m}$ in diameter. The peaks in the PL spectra at $20 \mathrm{~K}$ are ascribed to excitonic indirect transitions mediated by the emission of TA, LO, and TO phonons which provide the wave-vector $|\mathbf{k}|=0.85(2 \pi / a)$ along $\langle 100\rangle$, the positions of the $\Delta_{1}$ conduction band minima. Note, the PL signature labeled FE(TO $+\mathrm{LO})$ is attributed to free exciton annihilation in which the TO and LO phonon-assisted parts are not resolved. Hammond et al. ${ }^{10,11}$ have shown that these can be resolved with higher resolution at $2.1 \mathrm{~K}$. Such studies ${ }^{10}$ showed that the ratio of the intensities of the LO and TO signatures decreases from 0.3 at $2.1 \mathrm{~K}$ and approaches 0.1 by $10 \mathrm{~K}$. From the theoretical expression in Ref. 10 for the line shape, which

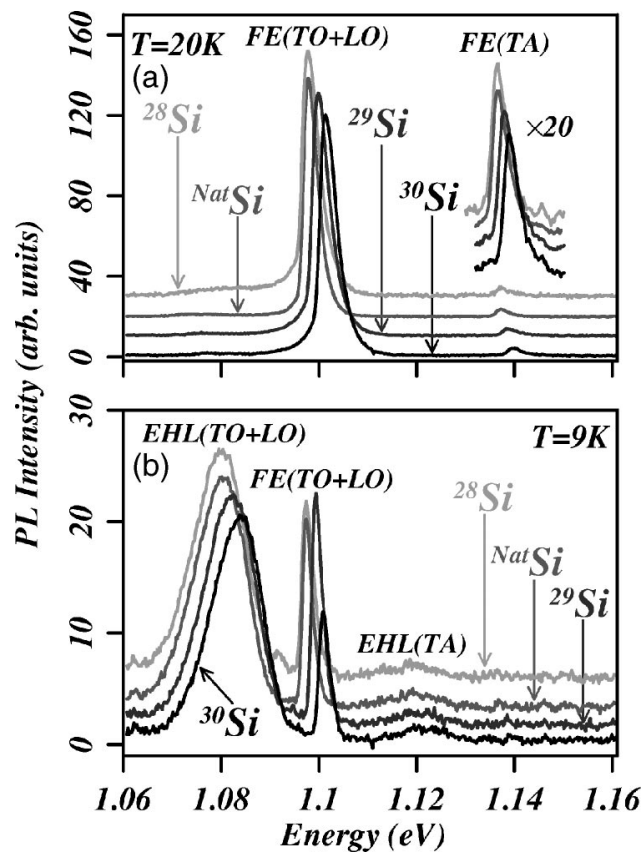

FIG. 1. Photoluminescence spectra of isotopically enriched and natural Si excited with an $\mathrm{Ar}^{+}$laser: (a) signatures of phononemission-assisted indirect excitonic transitions at $20 \mathrm{~K}$ and (b) at $9 \mathrm{~K}$, showing additional electron-hole-liquid signatures.
TABLE II. Positions of peaks observed in PL and WMT.

\begin{tabular}{lcccc}
\hline \hline & ${ }^{28} \mathrm{Si}(\mathrm{meV})$ & ${ }^{N a t} \mathrm{Si}(\mathrm{meV})$ & ${ }^{29} \mathrm{Si}(\mathrm{meV})$ & ${ }^{30} \mathrm{Si}(\mathrm{meV})$ \\
\hline & \multicolumn{4}{c}{} \\
FE(TA) & 1135.83 & 1135.98 & 1137.21 & 1138.28 \\
& \pm 0.10 & \pm 0.10 & \pm 0.10 & \pm 0.10 \\
FE(TO) & 1096.38 & 1096.70 & 1098.48 & 1100.00 \\
& \pm 0.10 & \pm 0.10 & \pm 0.10 & \pm 0.10 \\
EHL(TO) & $1088.5 \pm 0.3$ & $1088.7 \pm 0.3$ & $1090.7 \pm 0.3$ & $1092.0 \pm 0.3$ \\
& & $\mathrm{WMT}$ & & \\
TA $(n=1)$ & 1173.05 & 1173.15 & 1173.80 & 1174.39 \\
& \pm 0.05 & \pm 0.05 & \pm 0.05 & \pm 0.05 \\
LO $(n=1)$ & 1210.61 & 1210.67 & 1210.68 & 1210.74 \\
& \pm 0.05 & \pm 0.05 & \pm 0.05 & \pm 0.05 \\
LO $(n=2)$ & 1221.17 & 1221.18 & 1221.28 & 1221.39 \\
& \pm 0.10 & \pm 0.10 & \pm 0.10 & \pm 0.10 \\
TO $(n=1)$ & 1212.67 & 1212.72 & 1212.72 & 1212.74 \\
& \pm 0.05 & \pm 0.05 & \pm 0.05 & \pm 0.05 \\
TO $(n=2)$ & 1223.24 & 1223.23 & 1223.29 & 1223.34 \\
& \pm 0.10 & \pm 0.10 & \pm 0.10 & \pm 0.10 \\
\hline \hline
\end{tabular}

explicitly includes the sample temperature, it has been possible to deduce separately the positions of LO- and TOassisted peaks. The TA-assisted excitonic transition, labeled FE(TA), is significantly weaker. The peak positions of the TA-, LO-, and TO-assisted excitonic transitions are listed in Table II. In the PL spectra recorded at 9 K [Fig. 1(b)], strong and broad peaks labeled EHL(TO+LO) appear at energies lower than those of $\mathrm{FE}(\mathrm{TO}+\mathrm{LO})$; they correspond to TOand LO-assisted recombination radiation from an electronhole plasma, the electron-hole liquid. ${ }^{12}$ The feature labeled EHL(TA) is similarly the TA-assisted recombination radiation from EHL. The high energy thresholds for EHL(TO) are given in Table II.

The WMT spectra shown in Fig. 2 display the derivative signatures at $\left[E_{g x}(n)+\hbar \omega_{\mathbf{q}}\right]$ for the TA-, LO-, and TOassisted creation of indirect excitons in their $n=1$, i.e., ground, and $n=2$, i.e., the first excited, states ${ }^{13,14}$ for the various isotopically enriched ${ }^{28} \mathrm{Si},{ }^{29} \mathrm{Si}$, and ${ }^{30} \mathrm{Si}$ as well as for ${ }^{N a t} \mathrm{Si}$. The energies of these peaks are included in Table II.

Experiments on PL performed in the range $9 \mathrm{~K}$ to $20 \mathrm{~K}$ showed that the peak positions remained unchanged within experimental error $( \pm 0.1 \mathrm{meV})$; similarly, for WMT, the peak positions did not change in going from $5 \mathrm{~K}$ to $20 \mathrm{~K}$ within $\pm 0.05 \mathrm{meV}$. In view of this, it is justifiable to attribute the isotope related shifts entirely to effects related to zeropoint vibrations.

In Fig. 3 we present the $E_{g x}$, TO, LO, and TA energies as functions of $M$, deduced from the peak positions in Table II. The least squares fit follows $E_{g x}^{\infty}+C M^{-1 / 2}$ for the excitonic band gap $E_{g x}, E_{g x}^{\infty}$ being $(1213.8 \pm 1.2) \mathrm{meV}$, the value for $M=\infty$. A linear fit can be made over the small range of masses with a slope $\left(\partial E_{g x} / \partial M\right)$ of $1.01 \mathrm{meV} / \mathrm{amu}$. The phonon energies essentially obey $C^{\prime} M^{-1 / 2}$. The apparent mass independence of the TO-assisted $n=1,2$ excitons in Fig. 2 is accidental in that $C \approx-C_{\mathrm{TO}}^{\prime}$ as can be seen from a comparison of Figs. 3(a) and 3(c). The experiments also in- 


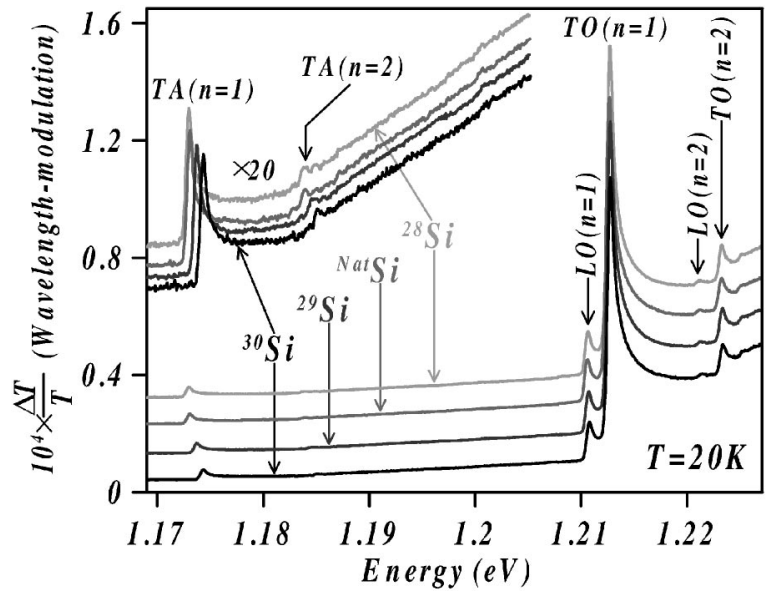

FIG. 2. Signatures of phonon-emission-assisted indirect excitonic transitions in isotopically enriched $\mathrm{Si}$ and ${ }^{\mathrm{Nat}} \mathrm{Si}$ observed in wavelength-modulated transmission.

dicate that the separation of the $n=2$ and $n=1$ excitons is isotope mass independent. Thus, the excitonic binding energy appears to be independent of $M$ within experimental errors.

In Table III we display in the third column the total change in the indirect excitonic energy per atomic mass unit $\left(\partial E_{g x} / \partial M\right)_{T, P}$, which has emerged from the present data for $\mathrm{Si}$ and compare it with those measured for diamond ${ }^{15}$ and Ge. ${ }^{6}$ In the fourth column we show the contribution from the isotope related change in the atomic volume, $(D / V)(\partial V / \partial M)_{T, P}$, calculated using (i) the measured values of the lattice parameter as a function of $M$ and (ii) Eq. (2) expressed in terms of macroscopic parameters. The contribution from the electron-phonon interaction $\left(\partial E_{g x} / \partial M\right)_{T, V}$, shown in the fifth column, is then the difference between the values in columns III and IV according to Eq. (1). The last column gives the theoretical values in Zollner et al. ${ }^{18}$ and Lautenschlager et l. $^{22}$ It is interesting to note that $(D / V)(\partial V / \partial M)_{T, P}$ deduced in these two ways agree very
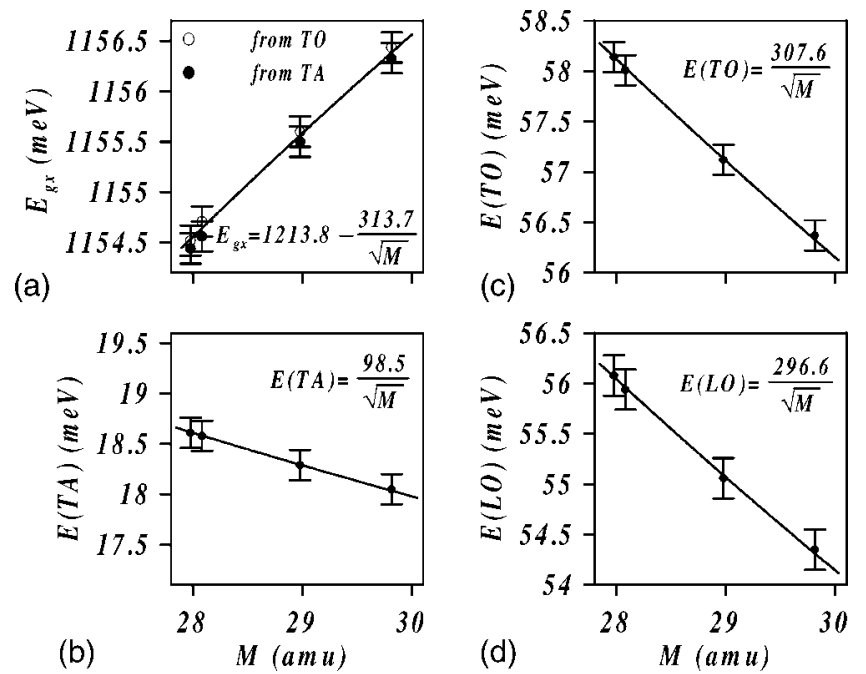

FIG. 3. The excitonic indirect band gap and the associated phonon energies as a function of $M$.
TABLE III. Isotope mass renormalization of the indirect band gap in diamond, $\mathrm{Si}$ and $\mathrm{Ge}$.

\begin{tabular}{|c|c|c|c|c|c|}
\hline & $\begin{array}{c}D \\
(\mathrm{meV})\end{array}$ & $\left(\frac{\partial E_{g x}}{\partial M}\right)_{T, P}$ & $\begin{array}{r}\frac{D}{V}\left(\frac{\partial V}{\partial M}\right)_{T, P} \\
(\mathrm{meV}\end{array}$ & $\left(\frac{\partial E_{g x}}{\partial M}\right)_{T, V}$ & $\left(\frac{\partial E_{g x}}{\partial M}\right)_{T, V}^{t h}$ \\
\hline $\mathrm{C}$ & $-2212^{\mathrm{a}}$ & $14.0^{\mathrm{b}}$ & $\begin{array}{l}\text { (i) } 1^{\mathrm{c}} \\
\text { (ii) } 0.8^{\mathrm{e}}\end{array}$ & $\begin{array}{l}13.0 \\
13.2\end{array}$ & $17.1^{\mathrm{d}}$ \\
\hline $\mathrm{Si}$ & $1410^{f}$ & $1.01^{\mathrm{g}}$ & $\begin{array}{l}\text { (i) }-0.123^{\mathrm{h}} \\
\text { (ii) }-0.114^{\mathrm{e}}\end{array}$ & $\begin{array}{l}1.133 \\
1.124\end{array}$ & $1.25^{\mathrm{i}}$ \\
\hline $\mathrm{Ge}$ & $-3800^{f}$ & $0.36^{\mathrm{j}}$ & $\begin{array}{l}\text { (i) } 0.096^{\mathrm{k}} \\
\text { (ii) } 0.085^{\mathrm{e}}\end{array}$ & $\begin{array}{l}0.264 \\
0.275\end{array}$ & $0.374^{\mathrm{d}}$ \\
\hline
\end{tabular}

${ }^{\text {aReference } 16 .}$

${ }^{b}$ Reference 15 .

${ }^{\mathrm{c}}$ Reference 17.

${ }^{\mathrm{d}}$ Reference 18 .

${ }^{\mathrm{e}}$ Reference 19.

fReference 20 .

gPresent results.
${ }^{\mathrm{h}}$ Reference 21.
${ }^{\mathrm{i}}$ Reference 22.
${ }^{\mathrm{j}}$ Reference 6.
${ }^{\mathrm{k}}$ Reference 9.

well. Equally noteworthy is the opposite signs of $(D / V)(\partial V / \partial M)_{T, P}$ for diamond and Ge on the one hand and $\mathrm{Si}$ on the other. The value for $\left(\partial E_{g x} / \partial M\right)_{T, V}$ deduced in this analysis for diamond and $\mathrm{Ge}$ are in reasonable agreement with the theoretical calculation of Zollner et al. ${ }^{18}$ and for $\mathrm{Si}$ with that in Lautenschlager et al. ${ }^{22}$

Collins et al., ${ }^{15}$ Davies et al., ${ }^{23}$ and Karaiskaj et al. ${ }^{24}$ have addressed the phenomenon of isotopic mass dependence of the indirect gap in diamond, $\mathrm{Ge}$ and $\mathrm{Si}$, respectively, on the basis of the energies of donor- or acceptorbound excitons observed in PL. In order to deduce the mass dependence of the indirect gap they assume the binding energy of the bound exciton to be independent of $M$. Parks et $a l .{ }^{6}$ have employed the combination of signatures in PL and modulated transmission and deduced both $E_{g x}$ and the energies of the associated phonons; this procedure in $\mathrm{Ge}$, as well as for $\mathrm{Si}$ in the present work, is free from the above assumption. It is gratifying to note that $\left(\partial E_{g x} / \partial M\right)_{T, P}$ in Refs. 6 and $23(\mathrm{Ge})$ and Ref. 24 and the present work $(\mathrm{Si})$ are in excellent agreement.

In Fig. 4 we display the WMT spectra where signatures labeled $\mathrm{TO}_{\mathrm{SO}}(n=1)$ and $\mathrm{TA}+\mathrm{O}(n=1)$ appear for ${ }^{28} \mathrm{Si},{ }^{29} \mathrm{Si}$, and ${ }^{30} \mathrm{Si}$. These features for ${ }^{\mathrm{Nat}} \mathrm{Si}$ were observed by Nishino et al. ${ }^{14}$ who attributed the former to a TO-assisted creation of an indirect exciton associated with the spin-orbit split $\Gamma_{7}^{+}\left(p_{1 / 2}\right)$ zone center valence band maximum and the $\Delta_{1}$ conduction band minima. The basis for this interpretation is the magnitude of the spin-orbit splitting $\Delta_{\text {SO }}$ being $44.1 \mathrm{meV}$ determined from the Lyman spectrum of boron acceptors in Si by Zwerdling et al. ${ }^{25}$ in exact coincidence with that from WMT. ${ }^{14}$ The feature labeled TA $+\mathrm{O}(n=1)$ is due to the indirect excitonic transition from the $\Gamma_{8}^{+}$valence band assisted by the simultaneous emission of TA and zone center optical phonon $(\mathrm{O})$. The $\mathrm{TO}_{\mathrm{SO}}(n=1)$ signatures in ${ }^{28} \mathrm{Si},{ }^{29} \mathrm{Si}$, and ${ }^{30} \mathrm{Si}$ coincide at $(1.258 \pm 0.002) \mathrm{eV}$, just as those of $\mathrm{TO}(n=1)$ in Fig. 2 do at (1.212 72 \pm 0.000 05) eV. From these observations we conclude that, within experimental errors, $\Delta_{\mathrm{SO}}$ is independent of $M$, as expected from theory in the first approximation. 


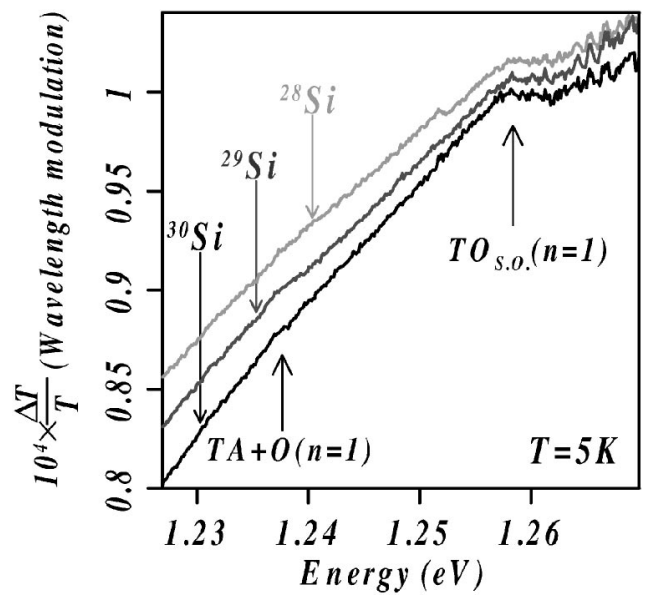

FIG. 4. The spin-orbit split indirect excitonic transitions in ${ }^{28} \mathrm{Si}$, ${ }^{29} \mathrm{Si}$, and ${ }^{30} \mathrm{Si}$.

It is equally interesting to note that the work function for the formation of EHL, deduced from the line-shape analysis of its relatively broad signature in PL following Hammond et $a l .{ }^{11}$ and $L o,{ }^{26}$ is $(8.0 \pm 0.3) \mathrm{meV}$, independent of $M$.
The results obtained in the present investigation for $\mathrm{Si}$, together with the earlier work for $\mathrm{Ge}$ and $\mathrm{C}$, are examples of the unique scientific opportunities created by the access to isotopically controlled specimens of semiconductors. In Table III we have an instructive comparison of the change in their fundamental (indirect) energy gap $\left(E_{g x}\right)$ as a function of $M . E_{g x}(M)$ for $\mathrm{Si}$ and $\mathrm{Ge}$, analyzed in terms of Eq. (1), yield the purely "electronic" values for $M=\infty$, i.e., for the ideal semiconductor not influenced even by the zeropoint motion. For the specific phonons involved in the indirect transitions, the $M^{-1 / 2}$ dependence is also more sharply delineated in comparison with those deduced from two-phonon infrared and Raman spectroscopies. The order of magnitude larger value for $\left(\partial E_{g x} / \partial M\right)_{T, P}$ for diamond in comparison to those of $\mathrm{Si}$ and $\mathrm{Ge}$ underscores the dramatic effect of the significantly larger zero-point motion in diamond.

The work at Purdue and UCB received support from the National Science Foundation Grant No. DMR0102699 and Grant No. DMR0109844, respectively. In addition, the U. S. Department of Energy Contract No. DE-AC03-76SF00098 supported the work at LBNL.
${ }^{1}$ M. L. Cohen and J. R. Chelikowski, Electronic Structure and Optical Properties of Semiconductors (Springer-Verlag, Heidelberg, 1988).

${ }^{2}$ See, E. E. Haller, J. Appl. Phys. 77, 2857 (1995).

${ }^{3}$ T. R. Anthony and W. F. Banholzer, Diamond Relat. Mater. 1, 717 (1992)

${ }^{4}$ K. Takyu, K. M. Itoh, K. Oka, N. Saito, and V. I. Ozhogin, Jpn. J. Appl. Phys., Part 2 38, L1493 (1999); A. D. Bulanov, G. G. Devyatych, A. V. Gusev, P. G. Sennikov, H.-J. Pohl, H. Riemann, H. Schilling, and P. Becker, Cryst. Res. Technol. 35, 1023 (2000); T. Ruf, R. W. Henn, M. Asen-Palmer, E. Gmelin, M. Cardona, H.-J. Pohl, G. G. Devyatych, and P. G. Sennikov, Solid State Commun. 115, 243 (2000); F. Widulle, T. Ruf, M. Konuma, I. Silier, M. Cardona, W. Kriegseis, and V. I. Ozhogin, ibid. 118, 1 (2001).

${ }^{5}$ H. Y. Fan, Rep. Prog. Phys. 19, 107 (1956).

${ }^{6}$ C. Parks, A. K. Ramdas, S. Rodriguez, K. M. Itoh, and E. E. Haller, Phys. Rev. B 49, 14244 (1994).

${ }^{7}$ H. Y. Fan, Phys. Rev. 82, 900 (1951).

${ }^{8}$ E. Antončik, Czech. J. Phys., Sect. A 5, 449 (1955).

${ }^{9}$ M. Y. Hu, H. Sinn, A. Alatas, W. Sturhahn, E. E. Alp, H.-C. Wille, Yu. V. Shvyd'ko, J. P. Sutter, J. Bandaru, E. E. Haller, V. I. Ozhogin, S. Rodriguez, R. Colella, E. Kartheuser, and M. A. Villeret, Phys. Rev. B 67, 113306 (2003).

${ }^{10}$ R. B. Hammond, D. L. Smith, and T. C. McGill, Phys. Rev. Lett. 35, 1535 (1975)

${ }^{11}$ R. B. Hammond, T. C. McGill, and J. W. Mayer, Phys. Rev. B 13, 3566 (1976).

${ }^{12}$ For an excellent review, see J. P. Wolfe and C. D. Jeffries, in Electron-hole Droplets in Semiconductors, edited by C. D. Jeffries and L. V. Keldysh (North-Holland, Amsterdam, 1983), p. 431.
${ }^{13}$ K. L. Shaklee and R. E. Nahory, Phys. Rev. Lett. 24, 942 (1970).

${ }^{14}$ T. Nishino, M. Takeda, and Y. Hamakawa, Solid State Commun. 12, 1137 (1973); 14, 627 (1974).

${ }^{15}$ A. T. Collins, S. C. Lawson, G. Davies, and H. Kanda, Phys. Rev. Lett. 65, 891 (1990).

${ }^{16}$ P. J. Dean and P. A. Crowther, in Radiative Recombination in Semiconductors, edited by C. Benoit a la Guillaume (Dunod, Paris, 1965), p. 103.

${ }^{17}$ H. Holloway, K. C. Hass, M. A. Tamor, T. R. Anthony, and W. F. Banholzer, Phys. Rev. B 44, 7123 (1991).

${ }^{18}$ S. Zollner, M. Cardona, and S. Gopalan, Phys. Rev. B 45, 3376 (1992).

${ }^{19}$ Computed using Eq. (2) with parameters from Landolt-Börnstein, New Series, Group III: Crystal and Solid State Physics, edited by O. Madelung, M. Schulz, and H. Weiss (Springer-Verlag, Heidelberg, 1982), Vol. 17 a.

${ }^{20}$ U. Schmid, N. E. Christensen, and M. Cardona, Solid State Commun. 75, 39 (1990).

${ }^{21}$ H.-C. Wille, Yu. V. Shvyd'ko, E. Gerdau, M. Lerche, M. Lucht, H. D. Rüter, and J. Zegenhagen, Phys. Rev. Lett. 89, 285901 (2002).

${ }^{22}$ Calculated from Fig. 5 in P. Lautenschlager, P. B. Allen, and M. Cardona, Phys. Rev. B 31, 2163 (1985). We thank M. Cardona for bringing this reference to our attention.

${ }^{23}$ G. Davies, E. C. Lightowlers, K. Itoh, W. L. Hansen, E. E. Haller, and V. Ozhogin, Semicond. Sci. Technol. 7, 1271 (1992).

${ }^{24}$ D. Karaiskaj, M. L. W. Thewalt, T. Ruf, M. Cardona, and M. Konuma, Solid State Commun. 123, 87 (2002).

${ }^{25}$ S. Zwerdling, K. J. Button, B. Lax, and L. M. Roth, Phys. Rev. Lett. 4, 173 (1960).

${ }^{26}$ T. K. Lo, Solid State Commun. 15, 1231 (1974). 